\title{
Digital microfluidics with pressure-based actuation
}

\author{
Elewout Hallynck and Peter Bienstman, Member, IEEE
}

\begin{abstract}
One of the key issues in biosensors is the time it takes for biomolecules in a solution to reach and bind to the sensor surface (particularly in low-concentration analytes). We present a novel flow scheme without microfluidic channels for labelfree biosensors to decrease the delivery time of biomolecules. By designing the biosensor in such a way that it becomes a membrane with holes, we can apply a droplet on it and push or pull it through the membrane by means of a pressure difference. Contrary to traditional microfluidics in e.g. flow cells where the analyte flows over the sensor, the flow is now directed through the sensor. We have implemented this scheme in Silicon-on-Insulator biosensors and have demonstrated in a first proof-of-principle experiment an improvement in delivery time of at least a factor of 3.
\end{abstract}

Index Terms-Silicon-on-Insulator, biosensor, membrane, digital microfluidics, pressure.

\section{INTRODUCTION}

B EFORE a drug is allowed on the consumer market, a process that takes up to tens of years can precede it [1]. At the start of this process, before testing on live subjects takes place, the potential drug has to be tested for several important criteria: is it stable, is it effective, is it toxic to some cells? Extensive studies take place to give a well-founded answer to all of these questions and one of the key instruments in providing those answers are biosensors.

Biosensors are becoming increasingly more sensitive up to the point where single molecules and fM concentrations can be detected [2]. However, some authors suggest that the fundamental LOD of some of these sensors has not yet been reached or that the time it takes to detect a single molecule can be greatly improved by optimising the transport mechanism of the target molecules dissolved in the analyte [3], [4]. While introduction of a laminar flow can increase analyte delivery considerably compared to pure diffusion, it is still not sufficient for these low-concentration analytes. In an attempt to further enhance the transport mechanism, several improved microfluidics configurations have been investigated [3]. Hofmann [5] proposes the introduction of a secondary flow that does not contain any target molecules and simply compresses the primary analyte flow. Huang et al [6] proposed directing a flow through a photonic crystal which acts as a biosensor. However, in their case a traditional microfluidic channel was still used.

In this work we will focus on a similar concept like that of Huang but without a microfluidic channel: consider a droplet of analyte on a biosensor with holes where we want the target molecules to bind at the walls of the holes (see Fig.

E. Hallynck and P. Bienstman are with the Photonics Research Group in the Department of Information Technology, Ghent University, Belgium.

E-mail: elewout.hallynck@intec.ugent.be

Manuscript received MONTH DAY, YEAR; revised MONTH DAY, YEAR.

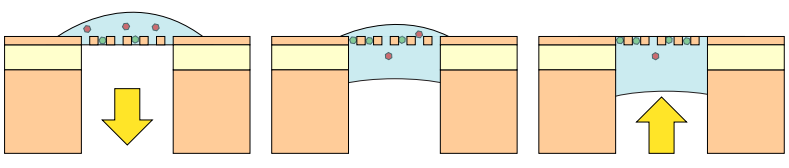

Fig. 1. By applying underpressure at the bottom of the liquid droplet, the biomolecules are transported through the sensor membrane. The droplet can be pushed back by applying overpressure. This cycle can be repeated several times to ensure most biomolecules have bonded in the membrane holes.

1). Gravity will not be able to sustain a flow by itself since it is counteracted by surface tension of the liquid. However, it is possible to initiate a flow by creating a pressure gradient over the droplet: creating an underpressure at the bottom of the droplet will cause the analyte containing the target molecules to be sucked through the openings. The target molecules will be brought in close contact with the receptor molecules with which they can bind. If we remove the pressure gradient, the droplet will be hanging on the other side of the membrane (i.e. the bottom). Since not all target molecules will have bonded to receptor molecules yet, we can push the droplet back through the openings by applying an overpressure at the bottom. The target molecules again pass closely to the receptor molecules and have a good chance at binding. If we stop the pressure gradient after a certain time, the droplet will be on top of the membrane once again. We can repeat this cycle several times to ensure most biomolecules have bonded in the membrane holes after some time. This concept has been demonstrated before by PamGene [7] in an application using different materials, length scales and transduction mechanisms. The advantages over the work of Huang [6] is that there is no need to encapsulate the sensor in a flow cell, saving on packaging costs and improving reliability (due to the high pressures involved in microfluidics, some flow cells tend to leak after some time), and that small volumes can easily be used since they are applied directly on the sensor and do not need to be transported through channels where in some cases target molecules can stick to the walls.

\section{MICROFLUIDICS THEORY}

The device we will use as an optical sensor is an integrated Mach-Zehnder interferometer (MZI). By underetching one of its arms in addition to creating a through-substrate via, we create a small membrane through which the flow can pass. Binding of biomolecules will cause a phase shift which is translated in the output spectrum of the device and can eventually be measured.

When working with structures with dimensions in the order of microns, surface forces become dominant over volume forces. When a droplet is applied to the sensor surface, it 
will encounter the waveguide trenches on both sides of the MZI arm which act as rectangular channels. The dimensions of these channels that we consider here are $2 \mu \mathrm{m}$ by $50 \mu \mathrm{m}$. We will examine the cases of a hydrophilic and hydrophobic surface as shown in Fig. 2. If we now consider the interface liquid-air in the trenches, we see that the liquid surface is curved. Since it requires energy to curve a surface, we can associate a pressure drop with the interface, the so-called Young-Laplace pressure drop $\Delta p_{\text {surf }}$ given by $\left(\frac{1}{R_{\phi}}+\frac{1}{R_{\theta}}\right) \gamma$ [8]. Here, $R_{\phi}$ and $R_{\theta}$ are the radii of curvature in the two directions and $\gamma$ is the surface tension in $\mathrm{N} / \mathrm{m}$. If we assume that we are dealing with either extreme hydrophilic of hydrophobic materials we can state that the radius of curvature is equal to the channel dimensions, divided by two. From the formula for the pressure drop it is clear that the dominant radius will be that of the smallest channel dimension, i.e. $2 \mu \mathrm{m}$. By convention we assume that with a positive curvature, a positive pressure is associated so we see that in the hydrophilic case, the pressure in the liquid at the interface is lower than that in the gas (and vice versa for the hydrophobic case). Considering now the hydrophilic case, the pressure in the liquid at the interface is defined as $p_{0}-\Delta p$. However, hydrostatic laws dictate that at that same point the pressure should also be $p_{0}^{*}+\rho g H$ with $\rho$ the density of the liquid, $g$ the gravitational constant and $H$ the local height of the droplet. Since the droplet at the top side is assumed to be quite large and the surface extremely hydrophilic, the curvature and associated pressure drop will be very small. Therefore, we can approximate $p_{0} \approx p_{0}^{*}$. The pressure inequality due to hydrostatics and surface tension, will push the liquid down the channel, driven by a pressure $\left(p_{0}^{*}+\rho g H\right)-\left(p_{0}-\Delta p\right)$. For water, with a surface tension $\gamma=72.9 \mathrm{~mJ}$, the pressure drop in this case amounts to $7.58 \times 10^{4} \mathrm{~Pa}$. In comparison, the hydrostatic pressure build-up in a droplet with $1 \mathrm{~mm}$ height is $10 \mathrm{~Pa}$, almost 4 orders of magnitude smaller. Due to the hydrophilic nature of the material, the liquid will spread itself out over the material. In practice, the channel dimensions will become larger (as we will see later). Although the magnitude of the pressure drop will decrease with increasing channel dimensions, the liquid will continue to propagate until an equilibrium is reached at the substrate side where a large curvature radius will be obtained. For the hydrophobic case, the situation is less favourable since there, we would have to counteract the large pressure drop in order to pull the liquid through the channels. It is therefore imperative that we work with hydrophilic materials. Silicon is naturally a hydrophilic material (with a contact angle of $22^{\circ}$ for water) and can be rendered even more hydrophilic by oxygen plasma treatment (with a contact angle of practically zero).

Once the equilibrium is reached, we need to apply pressure to pull the remainder of the droplet on top through the membrane. While in conventional fluidics, a constant pressure implies a constant flow, this is not the case when surface tension is involved. If we apply a small pressure gradient, the droplet at the substrate side will increase in size (using liquid from the top side of the device), thereby increasing its effective radius and Young-Laplace pressure drop. Once the

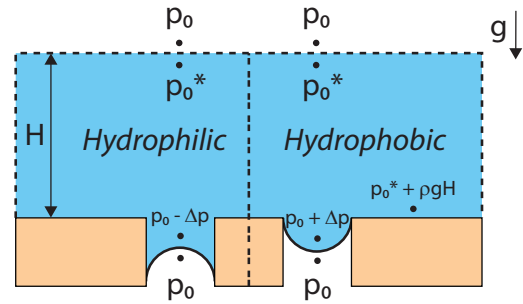

Fig. 2. Relevant pressures for a droplet in micro-channels for hydrophilic and hydrophobic case.

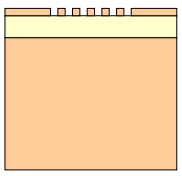

Patterning in SO
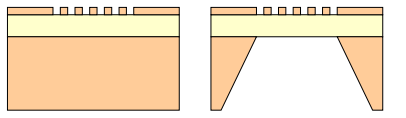

$\square$ Silicon $\quad \square$ Silicon dioxide

Substrate thinning Substrate etch
Fig. 3. The SOI dies are thinned by ePIXfab after which we locally etch the substrate and remove the buried silicon dioxide layer.

pressure drop is large enough to counter the applied pressure, the flow will stop. However, for a typical droplet of around $5 \mu l$, we can calculate that the pressure drop at the liquid-airinterface at the substrate side is in the order of tens of $\mathrm{Pa}$; the pressure gradient we will apply in our experiments is $10 \mathrm{kPa}$ so we can be quite certain that all liquid will have flown from the top to the substrate side before a new equilibrium is set.

To determine the flow rate for a given pressure gradient applied over the droplet, normally we can use the HagenPoiseuille theory which links the flow rate and pressure gradient through a geometry-dependent constant. One of the requirements for validity of this theory is that the channel length is long compared to the channel dimensions so that a steady-state regime of the velocity profile can be assumed. This is not the case here and using this theory would greatly underestimate viscous forces. Therefore, we turn to numerical calculations using COMSOL Multiphysics. For two $2 \mu \mathrm{m}$ by $50 \mu \mathrm{m}$ channels in parallel, the total simulated flow rate at a pressure gradient of $10 \mathrm{kPa}$ is about $0.7 \mu \mathrm{l} / \mathrm{s}$.

\section{FABRICATION}

Some of the requirements for a commercially viable biosensor is that it is low cost and capable of being produced in high volumes. A good candidate that is capable of delivering these requirements is Silicon-on-Insulator (SOI) in combination with Deep Ultraviolet (Deep UV) lithography. However, since we want to obtain thin membranes, we have to locally remove the substrate and buried oxide layer (see Fig. 3) from the devices received from ePIXfab. For etching the silicon substrate we have opted for the commonly used wet anisotropic etch through $\mathrm{KOH}$; for the silicon dioxide we used vapour HF etch since wet HF etch could cause stiction between the waveguides.

Since the standard substrate thickness of a SOI die is $725 \mu \mathrm{m}$, we opted to mechanically thin down the substrate first to $250 \mu \mathrm{m}$; otherwise, the hard mask material for the $\mathrm{KOH}$ etching process might not last long enough but more 


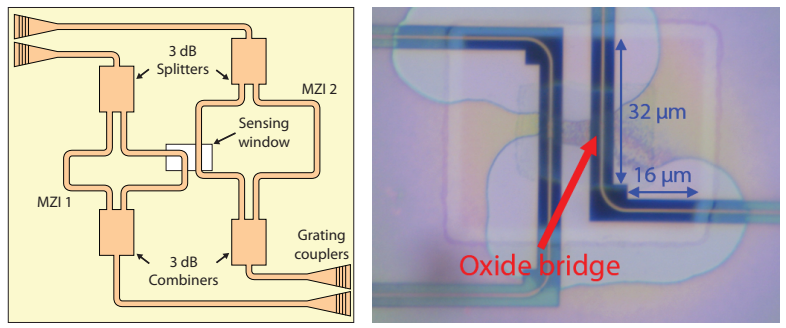

Fig. 4. Schematic overview of the entire layout (left) and a microscopic image after all fabrication steps (right) of the right arm of one MZI and the left arm of another one. The device in question is the right MZI.

importantly, the initial window size at the substrate size would need to be much larger (due to the anisotropic nature of the etching process). This implies that the density of several sensors on one chip (e.g. for multiplexing capabilities) has to be low since two windows in the hard mask cannot overlap. After thinning of the samples, we deposit $400 \mathrm{~nm} \mathrm{SiO}$ using plasma enhanced chemical vapour deposition (PECVD) at both sides and after that, $400 \mathrm{~nm}$ of PECVD silicon nitride (the $\mathrm{SiO}_{2}$ between the silicon and $\mathrm{Si}_{3} \mathrm{~N}_{4}$ acts as a buffer layer to minimise stress-induced effects due to the lattice mismatch between silicon and $S i_{3} N_{4}$ ). The layer stack at the device side acts as a protective layer for the light-guiding silicon structures in the 220-nm-thin silicon top layer while the layer stack at the bottom acts as a hard mask. After lithography using backside alignment (i.e. alignment markers at the device side are used to pattern the substrate side), the hard mask is locally etched using reactive ion etching (RIE). Since PECVD deposited $\mathrm{SiO}_{2}$ and $\mathrm{Si}_{3} \mathrm{~N}_{4}$ generally have a non-negligible pinhole density which could allow the $\mathrm{KOH}$ to damage our silicon structures, we require an extra protective coating at the device side. For this we use the commercially available ProTEK ${ }^{\circledR}$ B3 which is designed to withstand $\mathrm{KOH}$. With the layer stack finished, we put the sample in $20 \% \mathrm{KOH}$ at $79^{\circ} \mathrm{C}$ for 3 hours, where the 2- $\mu m$-thick buried silicon dioxide acts as an etch stop layer. After removal of the ProTEK ${ }^{\circledR}$ layer and cleaning of the sample, we perform vapour HF etching. The device side is here protected since it is electrostatically clamped to the chuck of the vapour HF tool. The underetched arm of the to be characterised device is pictured in the right of Fig. 4 (right). The total underetched arm length of the measured device is approximately $56 \mu \mathrm{m}$ (the bend radius is $5 \mu \mathrm{m}$ ). Note that in this device, non-uniformities occurred during vapour $\mathrm{HF}$ etching which resulted in a small silicion dioxide bridge attached to the underetched arm.

\section{Measurements}

\section{A. Set-up}

For characterising the sensor in the wavelength spectrum, we use a Santec TSL-510 tunable laser coupled to an optical fibre. Using a grating coupler [9], the light mode in the fibre is coupled to the TE mode of a silicon single mode waveguide in the top silicon layer. The light then enters the transducer (i.e. the Mach-Zehnder interferometer) and is coupled out again using another grating coupler. The light is collected using

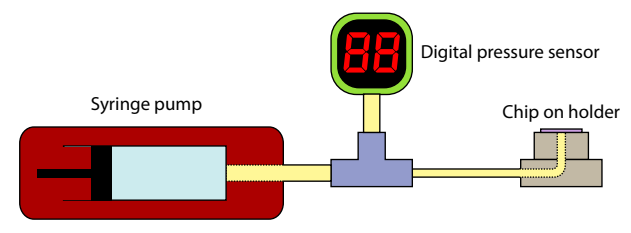

Fig. 5. A pressure gradient is applied by using a piston and is monitored using a digital pressure sensor.

another optical fibre and fed to an HP 8153A Optical power meter. As illustrated in Fig. 5, we designed a special mechanical chuck with which we can apply a pressure difference whilst keeping the sample in position using mechanical clamping (not shown). The pressure difference is generated by changing the volume - by means of a piston - of a pressure circuit that is on one end closed by the membrane of our chip. The top side of the membrane is always subject to atmospheric pressure.

Since fast spectrum acquisitions are required, we set up the tunable laser in continuous sweep mode. Each wavelength step sends a trigger signal to a data acquisition module (NI USB-6212) that uses the trigger signal to sample the analogue output of the power meter. A 14-nm-window (corresponding to the free spectral range of the device) around a spectral dip at around $1530 \mathrm{~nm}$ is continuously monitored (with an average acquisition time of $15 \mathrm{~s}$ per sweep), the theoretical cosine response of the MZI is fit to it and the wavelength shift is determined.

\section{B. Results}

To characterise the above device, we measure the shift of a spectral feature when a $5 \mu l$ droplet containing $1.25 \mathrm{mg} / \mathrm{ml}$ bovine serum albumine (BSA) is added to a $5 \mu l$ droplet of phosphate buffered saline (PBS) at $\mathrm{pH}$ 8. This experiment is straightforward since no receptor molecules need to be applied to our sensor because BSA binds directly to silicon [10]. Before starting the experiment, we want to ensure that our sensor is in fact hydrophilic. The contact angle of blank silicon is $22^{\circ}$ [8] but HF treated silicon has a contact angle of $70^{\circ}$ [11]. Although this should pose no real problem since the surface is still hydrophilic, we give our sensors a 10minute oxygen plasma treatment. Not only does this reduce the contact angle to practically $0^{\circ}$ (i.e. full wetting), but it also acts as an extra cleaning step to remove organic contaminants.

A measurement is divided into several pump cycles: an underpressure of $10 \mathrm{kPa}$ is applied at the substrate side of the chip for $10 \mathrm{~s}$. After that, an overpressure of $10 \mathrm{kPa}$ is applied at the substrate side of the chip for the same number of seconds. The pressure is stabilised again (i.e. no pressure gradient) and the wavelength spectrum is measured 10 consecutive times. When this is done, a new pump cycle is started. Note that we do not perform measurements when there is either over- or underpressure. This is because the elasto-optic effect created by this pressure gradient would cause an additional shift of the spectral features which has to be decoupled from the biomeasurements.

Fig. 6 shows the wavelength shift of a spectral dip at 1530 $\mathrm{nm}$ in function of time for the same biosensor in two situations 


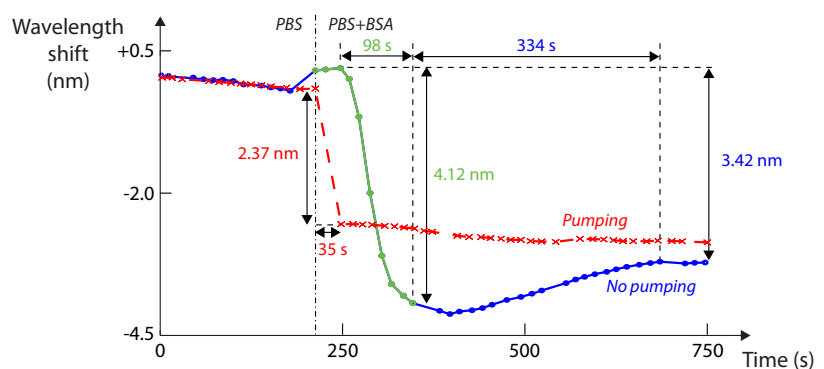

Fig. 6. When pumping (red, dashed curve) the binding time can be decreased by a factor of 3 , compared to no pumping (blue, solid curve). The green curve represents one measurement cycle of 10 data points.

(the sensor is reused after being cleaned using a Piranha solution): the dashed curve shows a measurement where the BSA droplet is introduced right before a new pump cycle starts and the solid curve shows a measurement where the BSA droplet is applied after pumping but before the wavelength measurements start, i.e. no subsequent pumping is performed to help the BSA reach the sensor. In both situations, before the BSA droplet is applied at $t \approx 210 \mathrm{~s}$, a pure PBS droplet is already present on the sensor.

When examining the binding event (i.e. transition from PBS to $\mathrm{PBS}+\mathrm{BSA}$ ), we notice a clear difference in the binding curves of both measurements. In the case where the BSA is applied after the pumping and before the 10 measurements of the current pump cycle, we see a typical binding curve (blue and green, solid curve) that starts off steeply and gradually reaches its peak value. In the other case, where the BSA is introduced and we immediately pump, we do not see this binding curve and immediately go into saturation (red, dashed curve). This is indeed the effect that we wanted to prove: by creating a flow path through the sensor (latter case), binding of the biomolecules occurs quicker than if we would have to wait for diffusion (former case). When quantitatively comparing both configurations, we see that the time it takes for binding with pumping is about $35 \mathrm{~s}$ (which is here limited by the pump duration and can be reduced upon optimisation) while the time it takes for the binding curve to reach its maximum is $98 \mathrm{~s}$, almost 3 times as slow. However, when examining the first case further, we see that there appears to be an overshoot between $t=250 \mathrm{~s}$ and $t=750 \mathrm{~s}$ in the binding curve (another overshoot before $t=250 \mathrm{~s}$ is caused by mechanical pumping effects). This phenomenon has also been observed by Glazer [12] in the binding behaviour of oligonucleotides to their receptor molecules where they explain that in some cases, two molecules bind to one probe, creating a metastable state. Eventually one of the molecules will desorb explaining the relaxation of the overshoot peak. Although we do not work with probes here but rely on direct binding of BSA to silicon, it is possible that the overshoot behaviour in our case can be attributed to a similar effect although a further, thorough analysis of the chemical reactions is required to be certain of this. It is interesting to note that in the case of pumping, this metastable state does not occur. If we also take into account the relaxation time for this overshoot, we see that in the case of no pumping it takes approximately 10 times longer to reach saturation. The difference in amount of shift between the two measurements is probably caused by temperature, mechanical effects or the fact that multilayers of BSA have formed; we have been unable to prove this in this proof-ofprinciple experiment. There is no apparent reason to state that using the pump delivers less biomolecules to the sensor surface as in both measurements, the sensor is eventually saturated.

\section{CONCLUSIONS}

We have characterised a Mach-Zehnder interferometerbased biosensor and were able to show that it is possible to achieve faster binding (in this case at least 3 times faster or 10 times if we include the overshoot relaxation time) of proteins to the biosensor using a flow through the biosensor. It should however be noted that the above experiment is a proof of concept and there is still room for improvement on several levels, e.g.: mechanically more robust structures, temperature controlled measurements, using TM-polarised light and lowconcentration binding, especially involving proper surface chemistry.

\section{ACKNOWLEDGMENT}

The authors would like to thank imec and the Belgian Science Policy Office, under grant IAP P7-35 photonics@be, for funding. Additional thanks to prof. J. Vierendeels for the discussions on fluidics, P. Guns for fabricating the device holder and T. Claes for the MZI designs.

\section{REFERENCES}

[1] PhRMA, "Pharmaceutical Research and Manufacturers of America." [Online]. Available: http://www.phrma.org/

[2] A. M. Armani, R. P. Kulkarni, S. E. Fraser, R. C. Flagan, and K. J. Vahala, "Label-free, single-molecule detection with optical microcavities." Science, vol. 317, pp. 783-7, Aug. 2007.

[3] P. E. Sheehan and L. J. Whitman, "Detection Limits for Nanoscale Biosensors," Nano Letters, vol. 5, no. 4, pp. 803-807, 2005.

[4] T. M. Squires, R. J. Messinger, and S. R. Manalis, "Making it stick: convection, reaction and diffusion in surface-based biosensors," Nature Biotechnology, vol. 26, no. 4, pp. 417-26, Apr. 2008.

[5] O. Hofmann, G. Voirin, P. Niedermann, and A. Manz, "ThreeDimensional Microfluidic Confinement for Efficient Sample Delivery to Biosensor Surfaces. Application to Immunoassays on Planar Optical Waveguides," Analytical Chemistry, vol. 74, pp. 5243-5250, 2002.

[6] M. Huang, A. A. Yanik, T.-Y. Chang, and H. Altug, "Sub-wavelength nanofluidics in photonic crystal sensors," Optics Express, vol. 17, no. 26, pp. 24 224-24 233, Dec. 2009.

[7] PamGene, "PamStation 12." [Online]. Available: https://www.pamgene.com/en/pamstation-technology.htm

[8] H. Bruus, Theoretical Microfluidics. Oxford University Press, 2008.

[9] D. Taillaert, W. Bogaerts, P. Bienstman, T. F. Krauss, P. V. Daele, I. Moerman, S. Verstuyft, K. D. Mesel, and R. Baets, "An Out-of-Plane Grating Coupler for Efficient Butt-Coupling Between Compact Planar," IEEE Journal of Quantum Electronics, vol. 38, no. 7, pp. 949-955, 2002.

[10] R. Desai and R. J. Richards, "The adsorption of biological macromolecules by mineral dusts," Environmental Research, vol. 16, pp. 44964, Jul. 1978.

[11] S. Franssila, Introduction to Microfabrication. Wiley, 2010.

[12] M. Glazer, J. a. Fidanza, G. H. McGall, M. O. Trulson, J. E. Forman, A. Suseno, and C. W. Frank, "Kinetics of oligonucleotide hybridization to photolithographically patterned DNA arrays," Analytical Biochemistry, vol. 358, no. 2, pp. 225-38, Nov. 2006. 